\section{Strategies for Automated Control of the Bioproduction of Mcl-PHA Biopolymers}

\author{
P. Hrnčiřík, ${ }^{*}$ J. Náhlík, and J. Mareš \\ Department of Computing and Control Engineering, \\ University of Chemistry and Technology, Prague, \\ Technická 5, 16628 Praha 6, Czech Republic
}

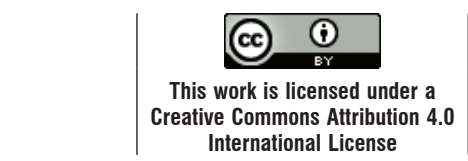

doi: 10.15255/CABEQ.2016.898

Original scientific paper Received: April 29, 2016 Accepted: July 21, 2017

Medium-chain-length polyhydroxyalkanoates (mcl-PHAs) are polyesters synthesized by numerous bacteria as storage material. Despite being promising candidates for biodegradable materials of industrial interest and environmental value, their usage is still rather limited because of high production costs. One of the areas with considerable potential for further improvements is control of the production process. This paper deals with the experimental work related to the design of control strategies for mcl-PHA biopolymer production process (Pseudomonas putida KT2442 fed-batch cultivations). For this bioprocess, a set of five control strategies (two main and three auxiliary strategies) have been proposed, together with the proper sequence of their switching during the fedbatch part of the production process. The application of these strategies with octanoic acid as a sole carbon source resulted in intracellular PHA content (max. mass fraction $65 \%$ of mcl-PHA in cell dry mass $\left(\mathrm{g} \mathrm{g}^{-1}\right)$ and PHA productivity (max. $0.89 \mathrm{~g} \mathrm{~L}^{-1} \mathrm{~h}^{-1}$ ) comparable to the best results reported in the literature for this type of strain and carbon substrate.

Key words:

fermentation, process control, biopolymer production, fed-batch cultivation

\section{Introduction}

Polyhydroxyalkanoates (PHAs) are microbial polyester polymers synthesized by numerous prokaryotes as carbon and energy storage material. These polymers are deposited intracellularly in the form of inclusion bodies and can amount up to $90 \%$ of cell dry mass. PHAs belong to promising candidates for biodegradable plastics and elastomers of industrial interest and environmental value. Potential fields of their application include, e.g., drug delivery, bone replacement applications, etc.

PHAs are generally classified into two major classes: short-chain-length PHAs (scl-PHAs) with C3-C5 monomers, and medium-chain-length PHAs (mcl-PHAs) with C6-C14 monomers. At present, only a few PHAs, typically from the structurally less complex class of scl-PHAs, are industrially produced. Especially in the case of the more complex mcl-PHAs, the disadvantage of high production costs has prevented their wide use despite their promising properties ${ }^{1}$.

Even though fermentation processes were developed employing different kinds of bacteria to improve mcl-PHAs productivity ${ }^{2,3}$, there is still con-

"Corresponding author: fax: +420 220445053 and e-mail:

Pavel.Hrncirik@vscht.cz siderable room for improvement, including the area of process control.

Fermentation processes used for mcl-PHAs production are typically based on fed-batch cultivations capable of achieving high-cell density cultures in an efficient way. The key issue related to the design of such processes is the choice of suitable substrate feeding strategies to control key nutrient concentrations, being in most cases the carbon source. The ideal solution, i.e., control strategies based on a direct measurement of the particular key nutrient concentration, is in many cases not routinely feasible due to the limited availability of the necessary analytical devices (problems with time delay, cost issues, reliability $)^{4}$. A common solution to this problem is often the use of predetermined exponential feeding profiles, i.e., feeding recipes calculated in advance from historical data with the aim to achieve a prescribed cell growth rate. However, the main drawback of this approach is its lack of flexibility vis-a-vis unexpected process events, since it cannot compensate for process disturbances that may severely affect the cell physiology. More appropriate are therefore control strategies, where the key nutrient feeding is based on cell physiology, typically via one or several process variables that reflect the physiological state of the microbial culture, and are calculable or even directly measurable on-line. 
In the case of $P$. putida fermentations for biopolymer production, several nutrient feeding strategies (e.g. $\mathrm{CO}_{2}$ production rate based pulse feeding, cumulative $\mathrm{CO}_{2}$ production based continuous feeding, etc.) have been proposed in the literature ${ }^{5-9}$. However, most of these strategies are focused on the KT2440 strain, which is different in terms of mcl-PHAs production from the rifampicin-resistant variant KT2442 ${ }^{10}$, and used carbon sources other than octanoic acid, a substrate with specific properties and applications ${ }^{2,3,4,11}$.

Therefore, the focus of this study is on the development of feeding strategies for the production of mcl-PHA biopolymers by cultivation of the $P$. putida KT2442 strain, suitable for fully automated carbon source (octanoic acid) dosage during the fed-batch part of the process.

\section{Materials and methods}

\section{Microorganism and cultivation conditions}

The inocula ( $P$. putida KT2442) for fed-batch cultivations were prepared at $30{ }^{\circ} \mathrm{C}$ in shaking flasks in a rotary incubator (incubation duration: 16-18 h). Composition of the incubation medium per liter: $4.7 \mathrm{~g}\left(\mathrm{NH}_{4}\right)_{2} \mathrm{SO}_{4}, 0.8 \mathrm{~g} \mathrm{MgSO} \cdot 7 \mathrm{H}_{2} \mathrm{O}, 12 \mathrm{~g}$ $\mathrm{Na}_{2} \mathrm{HPO}_{4} \cdot 7 \mathrm{H}_{2} \mathrm{O}, 2.7 \mathrm{~g} \mathrm{KH}_{2} \mathrm{PO}_{4}, 3 \mathrm{~g}$ nutrient broth. Production medium for the fed-batch phase contained per litre: $4.7 \mathrm{~g}\left(\mathrm{NH}_{4}\right)_{2} \mathrm{SO}_{4}, 0.8 \mathrm{~g} \mathrm{MgSO}_{4} \cdot 7 \mathrm{H}_{2} \mathrm{O}$, $9 \mathrm{~g} \mathrm{Na} \mathrm{HPO}_{4} \cdot 7 \mathrm{H}_{2} \mathrm{O}, 2.03 \mathrm{~g} \mathrm{KH} \mathrm{PO}_{4}, 1 \mathrm{~g}$ octanoic acid and $10 \mathrm{~mL}$ trace element solution (composition per litre: $10 \mathrm{~g} \mathrm{FeSO} \cdot 7 \mathrm{H}_{2} \mathrm{O}, 3 \mathrm{~g} \mathrm{CaCl}, 2.2 \mathrm{~g}$ $\mathrm{ZnSO}_{4} \cdot \mathrm{H}_{2} \mathrm{O}, 0.5 \mathrm{~g} \mathrm{MnSO}_{4} \cdot 4 \mathrm{H}_{2} \mathrm{O}, 0.3 \mathrm{~g} \mathrm{H}_{3} \mathrm{BO}_{3}, 0.2 \mathrm{~g}$ $\mathrm{CoCl}_{2} \cdot 6 \mathrm{H}_{2} \mathrm{O}, \quad 0.15 \mathrm{~g} \quad \mathrm{Na}_{2} \mathrm{MoO}_{4} \cdot 2 \mathrm{H}_{2} \mathrm{O}, \quad 0.02 \mathrm{~g}$ $\left.\mathrm{NiCl}_{2}^{2} \cdot 6 \mathrm{H}_{2}^{2} \mathrm{O}, 1 \mathrm{~g} \mathrm{CuSO} \cdot 5 \mathrm{H}_{2}^{2} \mathrm{O}\right)$.

\section{Fermentation conditions and analytical procedures}

The fed-batch cultivations ( $P$. putida KT2442) were carried out under the following conditions (kept constant throughout all experiments): temperature $30{ }^{\circ} \mathrm{C}$, $\mathrm{pH}$-value 7 , stirrer speed $900 \mathrm{~min}^{-1}$, air flow rate $9.5 \mathrm{~L} \mathrm{~min}^{-1}$. Base $\left(14 \% \mathrm{NH}_{4} \mathrm{OH}\right)$ and acid $\left(17 \% \mathrm{H}_{3} \mathrm{PO}_{4}\right)$ solutions were added to the cultivation medium to control the $\mathrm{pH}$-value. The choice of $\mathrm{NH}_{4} \mathrm{OH}$ as base served the dual purpose of $\mathrm{pH}$ control and nitrogen source.

All cultivations were carried out in a 7-litre laboratory bioreactor (newMBR, Switzerland) at the Bioprocess Control Laboratory at the Department of Computing and Control Engineering of the Institute of Chemical Technology in Prague (ICT Prague). The bioreactor was equipped with an IMCS 2000 analogue control unit (temperature, $\mathrm{pH}$, stirrer speed, antifoam level, and airflow control), a programmable logic controller (Modicon Compact PC-E984-265, Schneider Electric, France) and the proprietary Biogenes II control system (based on Factory Suite 2000 software package, Wonderware, USA). The dissolved oxygen tension (DO) was measured by an oxygen probe (Mettler Toledo); the oxygen $\left(\mathrm{O}_{2}\right)$ and carbon dioxide $\left(\mathrm{CO}_{2}\right)$ concentrations in the off-gas were measured by SERVOMEX 1100 and 1440 analysers, respectively. The so obtained off-gas data were used for online calculation of $\mathrm{CO}_{2}$ production rate $(\mathrm{CPR})$ and $\mathrm{O}_{2}$ consumption rate (OUR). For the substrate supply to the bioreactor, a DP200 peristaltic pump (New Brunswick) was used. Control variables feeding rate, acid, base and antifoam addition were also recorded.

Biomass concentration in the bioreactor was determined off-line gravimetrically as cell dry mass. The intracellular PHA content was also determined gravimetrically. Biomass for PHA gravimetric determinations was prepared by centrifuging samples $(50 \mathrm{~mL})$. The pellet was washed three times with deionized water and lyophilised. PHA was extracted from the lyophilised cells by Soxhlet extraction with hot chloroform $(150 \mathrm{~mL})$ for $24 \mathrm{~h}$. Excess chloroform was then distilled off to obtain ca. $5 \mathrm{~mL}$ residue. PHA was subsequently precipitated in 10 volumes of cold methanol. The precipitated polymer was separated by decantation, the solvent evaporated to dryness, and the purified PHA was then weighed. The measurement error was estimated to be $\leq 10 \%$ for the biomass and PHA concentration data.

\section{Substrate feeding strategies}

In the following, a set of feeding strategies suitable for fully automated dosage of carbon source will be proposed for the studied mcl-PHAs production process. All strategies are based exclusively on standard online measured or calculated process variables.

\section{Strategy 1: Continuous feeding proportional to $\mathrm{CO}_{2}$ production rate (CPR_control)}

Strategy applicable primarily during the exponential growth phase, carbon substrate feeding rate is calculated by Eq. 1,

$$
F m(t)=k C P R \cdot C P R(t) \cdot V(t) / \rho_{-} O A
$$

where $F m(t)\left(\mathrm{mL} \mathrm{min}{ }^{-1}\right)$ is the carbon source (octanoic acid) feeding rate at culture time $t ; k C P R$ is the desired carbon source/ $\mathrm{CO}$ ratio (g octanoic acid added/g CO produced); $C P R(t)$ is the $\mathrm{CO}_{2}$ production rate $\left(\mathrm{g} \mathrm{L}^{-1} \mathrm{~min}^{-1}\right)$ at culture time $t ; V(t)$ is the liquid volume in the fermenter (L) at culture time $t$, and $\rho \_O A$ is the density of the octanoic acid $(0.910$ $\left.\mathrm{g} \mathrm{mL}^{-1}\right)$. 
Strategy 2: Continuous feeding proportional to $\mathrm{O}_{2}$ uptake rate (OUR_control)

Alternative strategy to Strategy 1, applicable primarily during the exponential growth phase, carbon substrate feeding rate is calculated by Eq. 2,

$$
F m(t)=k O U R \cdot O U R(t) \cdot V(t) / \rho_{-} O A
$$

where $F m(t)\left(\mathrm{mL} \mathrm{min}^{-1}\right)$ is the carbon source (octanoic acid) feeding rate at culture time $t ; k O U R$ is the desired carbon source $/ \mathrm{O}_{2}$ ratio ( $\mathrm{g}$ octanoic acid added/g $\mathrm{O}_{2}$ consumed); $O U R(t)$ is the $\mathrm{O}_{2}$ uptake rate $\left(\mathrm{g} \mathrm{L}^{-1} \mathrm{~min}^{-1}\right)$ at culture time $t ; V(t)$ is the liquid volume in the fermenter (L) at culture time $t$, and $\rho \_O A$ is the density of the octanoic acid $\left(0.910 \mathrm{~g} \mathrm{~mL}^{-1}\right)$.

\section{Strategy 3: Continuous feeding following a linear feeding profile}

An auxiliary strategy applicable upon the completion of the initial batch phase, at the beginning of the fed-batch part of cultivation in cases where the microbial culture's consumption and production rates are too low (OUR, CPR below approximately $0.5 \mathrm{~g} \mathrm{~L}^{-1} \mathrm{~h}^{-1}$, as determined experimentally in Cultivations 2 and 3 , see the section of the paper on cultivations in "Results and discussion") to safely operate strategies based on the consumption/production rates data (Strategy 1 and 2). Initial feeding rate is usually set at the value corresponding to the carbon source consumption rate attained in the batch phase, calculated online at the end of the batch phase by Eq. 3,

$$
R_{C-S U B, B A T C H}=\frac{V_{C-S U B, B A T C H}}{\Delta T_{B A T C H}}
$$

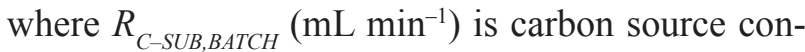
sumption rate in the batch phase; $V_{C-S U B, B A T C H}(\mathrm{~mL})$ is the volume of carbon substrate added at the start of the cultivation, and $\Delta T_{B A T C H}$ ( $\mathrm{min}$ ) is the time length of the batch phase. The initial feeding rate is then increased linearly, at a rate of approximately 25 percent per hour, until the microbial culture's consumption/production rates reach levels that allow safe switching to Strategy 1 or 2 .

\section{Strategy 4: Semicontinuous feeding using the reading of the dissolved oxygen as controlled variable (DO-based pulse feeding)}

This strategy represents an alternative to Strategy 3 , being also primarily applicable at the beginning of the fed-batch part of cultivation where the consumption and production rates are still relatively low compared to later cultivation stages. Strategy 4 is principally based on the detection of carbon source limitation from the time course of the DO measurement, i.e., a single dose of the carbon source is added to the fermenter each time the DO value increases by a given amount (typically in the range of 1 to $2 \%$ ) of its previous minimum value. The volume of the dose is calculated from the carbon source consumption rate in the batch phase (Eq. 3) by Eq. 4,

$$
D_{C-S U B}=R_{C-S U B, B A T C H} \cdot \Delta T
$$

where $D_{C-S U B}(\mathrm{~mL})$ is the volume of a single dose of the carbon substrate, and $\Delta T$ (min) is the desired initial time interval between two substrate additions (typically $10 \mathrm{~min}$ ). Since the time interval decreases gradually as the culture grows, the $D_{C-S U B}$ value can be increased accordingly throughout the operation of this feeding strategy. One possible scenario is to increase the $D_{C-S U B}$ value by $50 \%$ of its initial value each time the time interval decreases below $30 \%$ of the $\Delta T$ value.

\section{Strategy 5: Continuous feeding using the reading of the dissolved oxygen as controlled variable (PID controlled DO-stat)}

Strategy applicable primarily during the final phase of oxygen limitation with the carbon source feeding rate being manipulated by a standard proportional-integral-derivative controller in order to maintain the dissolved oxygen concentration around an appropriate set point (usually $20 \%$ sat.) to avoid carbon substrate overfeeding.

\section{Results and discussion}

The experimental work done within this study had two main objectives. Firstly, it aimed to test the proposed feeding strategies and verify the proper sequence of their switching during the fed-batch part of the cultivation. The second objective was to evaluate various ratios of the added carbon source to the amount of produced $\mathrm{CO}_{2}$ or consumed $\mathrm{O}_{2}$, respectively (control parameters of Strategy $1-$ $k C P R$ and $2-k O U R$ ), with regard to their effect on the mcl-PHAs production. For this purpose, the proprietary Biogenes II control system has been enhanced with functions for on-line calculation of real values of both these ratios, each in two variants (overall and time-window), see Eqs. 5-8.

$$
\begin{gathered}
\operatorname{Rat}_{C-\text { SOURCE } / C O_{2}}^{0 \rightarrow T}=\frac{\int_{0}^{T} F m(t) \mathrm{d} t}{\int_{0}^{T} C P R(t) \mathrm{d} t} \\
\operatorname{Rat}_{C-\text { SOURCE } / \mathrm{CO}_{2}}^{T-\triangle T \rightarrow T}=\frac{\int_{T-\Delta T}^{T} F m(t) \mathrm{d} t}{\int_{T-\Delta T}^{T} C P R(t) \mathrm{d} t}
\end{gathered}
$$




$$
\begin{aligned}
\operatorname{Rat}_{C-S O U R C E / O_{2}}^{0 \rightarrow T} & =\frac{\int_{0}^{T} F m(t) \mathrm{d} t}{\int_{0}^{T} \text { OUR }(t) \mathrm{d} t} \\
\operatorname{Rat}_{C-S O U R C E / O_{2}}^{T-\triangle T \rightarrow T} & =\frac{\int_{T-\Delta \mathrm{T}}^{T} F m(t) \mathrm{d} t}{\int_{T-\Delta \mathrm{T}}^{T} \text { OUR }(t) \mathrm{d} t}
\end{aligned}
$$

where $T(\mathrm{~min})$ is the current cultivation time, and $\Delta T(\min )$ is an arbitrary time window length chosen by a human operator supervising the cultivation process. These on-line calculations served as a support tool in the process of tuning Strategies 1 and 2, i.e., the choice of $k C P R$ or $k O U R$ values, respectively. The adopted experimental approach was to start from the ratio values attained in the initial batch phase of the first cultivation and then to gradually adjust the $k C P R$ or $k O U R$ values on the basis of obtained results. The specific setting of these parameters in individual fed-batch cultivations is described under each experiment.

\section{Cultivations}

In total, a series of five fed-batch cultivations (Cultivation $1-5$ ) were carried out under different carbon source feeding scenarios. All cultivations started with a batch phase (initial cell dry mass around $1.5 \mathrm{~g} \mathrm{~L}^{-1}$ ) using octanoic acid (initial concentration: $1 \mathrm{~g} \mathrm{~L}^{-1}$ ) as the sole carbon source. Following the initial batch phase, the same carbon source was supplied with a time-varying feeding rate calculated online using either a single feeding strategy or a sequence of several feeding strategies. Specifically, in Cultivations 1, 2, and 3, only Strategy 1 in combination with a manually set feeding rate was used, each cultivation was run with a different value of the $k C P R$ parameter. In the last two cultivations, a sequence of three strategies was used - in Cultivation 4 Strategies 3, 1, and 5, and in Cultivation 5 Strategies 4, 2, and 5 .

\section{Cultivation 1}

The initial batch phase lasted 6 hours. Immediately thereafter, the fed-batch phase was started using Strategy 1 (continuous feeding proportional to CPR) with the $k C P R$ parameter set to the value of $1.77 \mathrm{~g} \mathrm{~g}^{-1}$, calculated by Eq. 5 as the ratio of the added carbon source to the amount of produced $\mathrm{CO}_{2}$ in the initial batch phase $\left(\right.$ Rat $\left._{\text {C-SOURCE/Co }}^{0 \rightarrow 6}\right)$. At 22 $\mathrm{h}$, the strategy was stopped at the end of the exponential growth phase because of the approaching onset of oxygen limitation and emerging symptoms of possible carbon source overfeeding (DO around $35 \%$ sat. and stagnating, while carbon source feeding rate was increasing). After a short period with- out feeding $(1.5 \mathrm{~h})$, Strategy 1 was switched on again for the final phase of oxygen limitation with a lower $k C P R$ value $\left(1.64 \mathrm{~g} \mathrm{~g}^{-1}\right)$, calculated by Eq. 5 as the ratio of the added carbon source to the amount of produced $\mathrm{CO}_{2}$ over the entire cultivation duration up to that time instant $\left(R a t_{C-S O U R C E / C O_{2}}^{0 \rightarrow 23.5}\right)$. The cultivation ended at $28 \mathrm{~h}$ when the DO value reached $0 \%$ sat., with a final cell dry mass of $32.4 \mathrm{~g} \mathrm{~L}^{-1}$ and mass fraction $42 \%$ of mcl-PHA in cell dry mass $\left(\mathrm{g} \mathrm{g}^{-1}\right)$ (for comparison of results, see Table 1).

\section{Cultivation 2}

As in the first experiment, Strategy 1 was started right after the end of the batch phase (batch duration: 4 hours) with the $k C P R$ parameter set to a lower value than the value used in the final stage of Cultivation 1 ( $95 \%$ of $1.64 \mathrm{~g} \mathrm{~g}^{-1} \rightarrow 1.55 \mathrm{~g} \mathrm{~g}^{-1}$ ) in order to investigate its effect on the PHA production. At $8 \mathrm{~h}$, the strategy was stopped because of emerging symptoms of possible carbon source overfeeding (DO around $92 \%$ sat. and changing from stagnation to increase, while carbon source feeding rate was increasing). The most likely underlying cause for this event was the unstable perturbed character of the still relatively low CPR values (around $0.2 \mathrm{~g} \mathrm{~L}^{-1} \mathrm{~h}^{-1}$ ) at this early stage of the fedbatch part of the cultivation. After that, the carbon source feeding rate was set manually to maintain the carbon source added $/ \mathrm{CO}_{2}$ produced ratio within the range from 1.2 to $1.5 \mathrm{~g} \mathrm{~g} \mathrm{~g}^{-1}$. Strategy 1 was switched on again at $22 \mathrm{~h}$ at the beginning of the exponential growth phase with $k C P R$ set to $1.48 \mathrm{~g} \mathrm{~g}^{-1}$, calculated by Eq. 5 as the ratio of the added carbon source to the amount of produced $\mathrm{CO}_{2}$ over the entire cultivation duration up to that time instant $\left(\right.$ Rat $\left._{\mathrm{C}-\mathrm{SOURCE} / \mathrm{CO}_{2}}^{0 \rightarrow 22}\right)$. The cultivation ended at $34 \mathrm{~h}$ when the DO value reached $0 \%$ sat., with a final cell dry mass of $61.5 \mathrm{~g} \mathrm{~L}^{-1}$ and mass fraction $55 \%$ of mcl-PHA in cell dry mass $\left(\mathrm{g} \mathrm{g}^{-1}\right)$ (for comparison of results, see Table 1).

\section{Cultivation 3}

Similarly to the previous experiments, Strategy 1 was used from the end of the batch phase (batch duration: 5.5 hours) with the $k C P R$ parameter set to the same value as in the exponential growth phase of Cultivation $2\left(1.48 \mathrm{~g} \mathrm{~g}^{-1}\right)$. At $8.5 \mathrm{~h}$, a similar scenario occurred as in Cultivation 2, and the strategy was stopped because of emerging symptoms of possible carbon source overfeeding (DO around 90\% sat. and changing from stagnation to increase, while carbon source feeding rate was increasing). Again, the most likely cause was the unstable perturbed character of the still relatively low CPR values (around $0.3 \mathrm{~g} \mathrm{~L}^{-1} \mathrm{~h}^{-1}$ ) at this early stage of the fedbatch part of the cultivation. The carbon source 
Table 1 -Overview of experimental results related to mcl-PHAs production, cultivations $1-5$

\begin{tabular}{|c|c|c|c|c|c|c|c|}
\hline Cultivation & $\begin{array}{c}\mathrm{C} \text {-source } / \mathrm{CO}_{2} \\
\text { ratio - exp. } \\
\text { growth } \\
\text { phase }\left(\mathrm{g} \mathrm{g}^{-1}\right)\end{array}$ & $\begin{array}{l}\mathrm{C} \text {-source } / \mathrm{O}_{2} \\
\text { ratio - exp. } \\
\text { growth phase } \\
\qquad\left(\mathrm{g} \mathrm{g}^{-1}\right)\end{array}$ & $\begin{array}{l}\mathrm{C} / \mathrm{N} \text { ratio }- \\
\text { exp. growth } \\
\text { phase } \\
\left(\mathrm{mol} \mathrm{mol}^{-1}\right)\end{array}$ & $\mu\left(\mathrm{h}^{-1}\right)$ & $\begin{array}{l}\text { PHA content } \\
\text { as mass fraction } \\
\text { of mcl-PHA in } \\
\text { dry cell mass } \\
\text { (wt. } \%)\end{array}$ & $\begin{array}{c}\text { PHA } \\
\text { productivity } \\
\left(\mathrm{g} \mathrm{L}^{-1} \mathrm{~h}^{-1}\right)\end{array}$ & $\begin{array}{c}\text { Conversion } \\
\text { yield (C-source } \\
\text { to PHA) } \\
\left(\mathrm{g} \mathrm{g}^{-1}\right)\end{array}$ \\
\hline 1 & 1.77 & 1.50 & 17.2 & 0.18 & 42 & 0.50 & 0.26 \\
\hline 2 & 1.48 & 1.11 & 15.0 & 0.15 & 55 & 0.89 & 0.47 \\
\hline 3 & 1.30 & 1.03 & 15.9 & 0.12 & 65 & 0.83 & 0.54 \\
\hline 4 & 0.90 & 0.72 & 11.0 & 0.09 & 46 & 0.67 & 0.48 \\
\hline 5 & 1.82 & 1.48 & 20.5 & 0.18 & 41 & 0.57 & 0.22 \\
\hline
\end{tabular}

feeding rate was then adjusted manually to maintain the carbon source $/ \mathrm{CO}_{2}$ ratio within the range from 1.1 to $1.4 \mathrm{~g} \mathrm{~g}^{-1}$. Strategy 1 was switched on again at $20 \mathrm{~h}$ at the beginning of the exponential growth phase with $k C P R$ set to $1.3 \mathrm{~g} \mathrm{~g}^{-1}$, calculated by Eq. 5 as the ratio of the added carbon source to the amount of produced $\mathrm{CO}_{2}$ over the entire cultivation duration up to that time instant $\left(\operatorname{Rat}_{\mathrm{C}-\mathrm{SOURCE} / \mathrm{CO}_{2}}^{0 \rightarrow \text {. At }}\right.$ $30 \mathrm{~h}$, when the DO value reached $0 \%$ sat., Strategy 1 was stopped and the cultivation was continued for another 6 hours using manually adjusted carbon source feeding rate maintaining the DO value slightly above $0 \%$ sat. The cultivation ended at $36 \mathrm{~h}$ with a final cell dry mass of $42.3 \mathrm{~g} \mathrm{~L}^{-1}$ and mass fraction $65 \%$ of mcl-PHA in cell dry mass $\left(\mathrm{g} \mathrm{g}^{-1}\right)$, which was the highest mcl-PHAs content achieved in this study (for comparison of results, see Table 1). The time profiles of DO, Fms (cumulative volume of added octanoic acid), CPR and PHA concentration are shown in Figure 1.

\section{Cultivation 4}

Unlike previous experiments, in this cultivation (batch duration: $6 \mathrm{~h}$ ) in order to avoid the occurrence of carbon source overfeeding, Strategy 3 (linear feeding profile) was used for the start of the fedbatch part. Initial feeding rate was calculated after completion of the batch phase using Eq. $3(0.025$ $\mathrm{mL} \min ^{-1}$ ), and then linearly increased over the next 2 hours at the rate of $25 \%$ per hour. At $8 \mathrm{~h}$, when the CPR and OUR values reached levels around 0.4 $\mathrm{g} \mathrm{L}^{-1} \mathrm{~h}^{-1}$ ), a switch in feeding strategies was realized from Strategy 3 to Strategy 1, with the $k C P R$ parameter set to a considerably lower value than in previous experiments (approx. $50 \%$ of the initial value used in Cultivation $1 \rightarrow 0.9 \mathrm{~g} \mathrm{~g}^{-1}$ ) in order to investigate its effect on the PHA production. At 18 $\mathrm{h}$, upon the onset of oxygen limitation (DO around $20 \%$ sat. and decreasing), a switch in feeding strategies was realized from Strategy 1 to Strategy 5

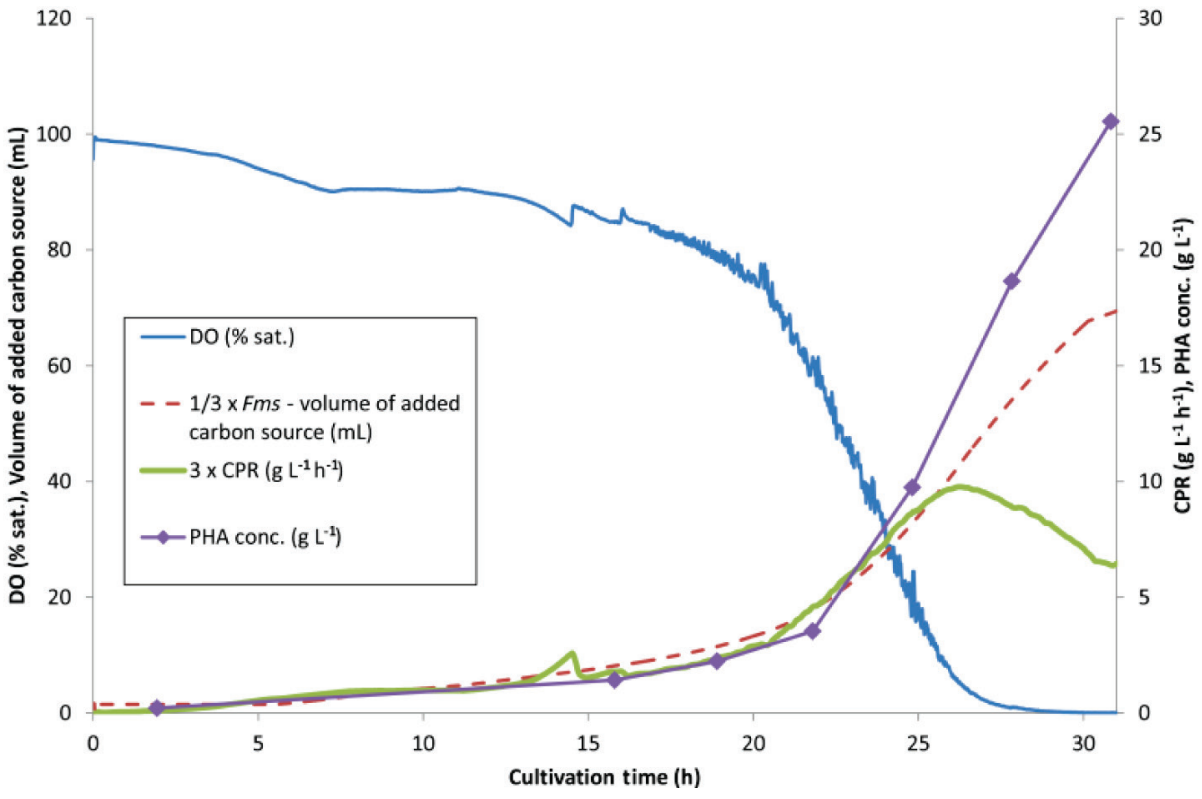

Fig. 1 - Cultivation $3(0-31$ h), carbon source feeding using Strategy 1 
(PID control of DO), with a DO set point at $20 \%$ sat. to avoid reaching the zero level of DO. In this regime, the cultivation was continued for another 15 hours. The cultivation ended at $33 \mathrm{~h}$ with a final cell dry mass of $26.9 \mathrm{~g} \mathrm{~L}^{-1}$ and mass fraction $40 \%$ of mcl-PHA in cell dry mass $\left(\mathrm{g} \mathrm{g}^{-1}\right)$ (for comparison of results, see Table 1).

\section{Cultivation 5}

The aim of the last experiment was to test alternative strategies - Strategy 4 (DO-based pulse feeding) for the start of the fed-batch part and Strategy 2 (continuous feeding proportional to OUR) for the phase of exponential growth. After the initial batch phase (duration: 5 hours), Strategy 4 was used to start the fed-batch. The volume of the single carbon source dose was calculated by Eqs. 3 and 4 with $\Delta T$ set to $10 \mathrm{~min}\left(D_{C-S U B}=0.2 \mathrm{~mL}\right)$. Single dose of the carbon source was then added to the fermenter each time the DO value increased by $2 \%$ of its previous minimum value. After 2 hours, the volume of the single dose was increased by $50 \%$ to $0.3 \mathrm{~mL}$, because the time interval between two subsequent carbon source additions decreased to $3 \mathrm{~min}$. At $9 \mathrm{~h}$, at the beginning of the exponential growth phase, when the CPR and OUR values reached levels around $0.8 \mathrm{~g} \mathrm{~L}^{-1} \mathrm{~h}^{-1}$, a switch in feeding strategies was realized from Strategy 4 to Strategy 2 (continuous feeding proportional to OUR), with the $k O U R$ parameter set to $1.48 \mathrm{~g} \mathrm{~g}^{-1}$, calculated by Eq. 8 as the ratio of the added carbon source to the amount of consumed $\mathrm{O}_{2}$ over the entire duration of the fedbatch phase up to that time instant, i.e., over the entire duration of Strategy $4\left(\mathrm{Rat}_{\mathrm{C}-\text { SOURCE/oo }}^{5 \rightarrow 9}\right)$. At 12 $\mathrm{h}$, upon the onset of oxygen limitation (DO around
$20 \%$ sat. and decreasing), a switch in feeding strategies was realized from Strategy 2 to Strategy 5 (PID control of DO), with a DO set point at $20 \%$ sat. to avoid reaching the zero level of DO. In this regime, the cultivation was continued for another 19 hours. The cultivation ended at $31 \mathrm{~h}$ with a final cell dry mass of $40.2 \mathrm{~g} \mathrm{~L}^{-1}$ and mass fraction $41 \%$ of mcl-PHA in dry cell mass $\left(\mathrm{g} \mathrm{g}^{-1}\right)$ (for comparison of results, see Table 1). The time profiles of DO, Fms (cumulative volume of added octanoic acid), OUR and PHA concentration are shown in Figure 2.

\section{Comparison of feeding strategies}

Experimental tests have proven the suitability of Strategies 1 and 2 for carbon source feeding during the exponential growth phase, maintaining the desired ratio between the feeding rate and CPR or OUR, respectively. Furthermore, for the studied process, the two feeding strategies were practically interchangeable, since the CPR/OUR ratio (known also as the respiration quotient RQ) in the exponential growth phase was more or less constant throughout all experiments at approx. 0.87 (see Figure 3, the constant RQ value of 0.87 corresponds to the slope value). On the other hand, their performance at the beginning of the fed-batch part before the exponential growth phase, and towards the end of cultivation, after the onset of oxygen limitation, was less satisfactory.

Specifically, the results from the first three experiments (Cultivation $1-3$ ) have shown that the use of Strategy 1 (continuous feeding proportional to $\mathrm{CPR}$ ) right from the end of the batch phase is problematic because of repeated occurrence of carbon source overfeeding. This undesirable phenome-

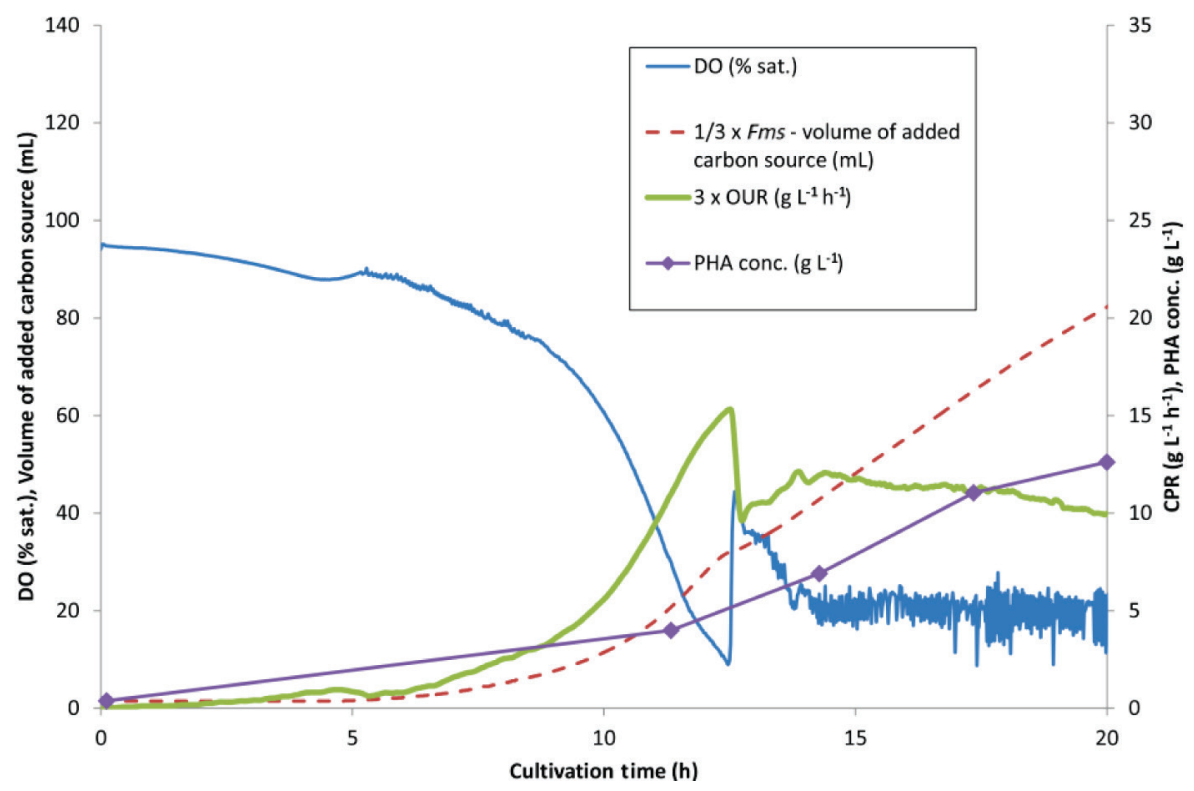

Fig. 2 - Cultivation $5(0-20 \mathrm{~h})$, carbon source feeding using sequence of strategies $(4,2,5)$ 


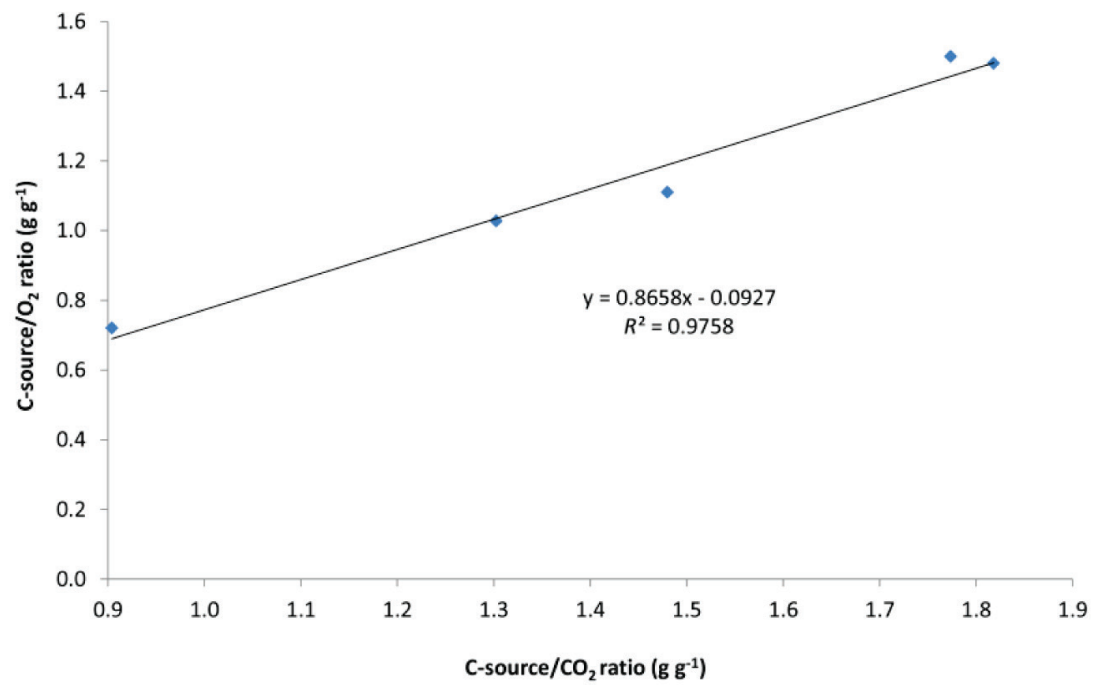

Fig. 3 - Relationship between $\mathrm{C}$-source/O $\mathrm{O}_{2}$ and $\mathrm{C}$-source/CO $\mathrm{C}_{2}$ ratios in the exponential growth phase, data from cultivations $1-5$

non is likely to occur in any application of this strategy (and Strategy 2) at the beginning of the fed-batch phase for feeding of carbon sources that are inhibitory at relatively low concentrations (e.g. octanoic acid inhibits $P$. putida at concentrations higher than $\left.4 \mathrm{~g} \mathrm{~L}^{-1}\right)^{3}$. Under these circumstances, perturbations in signals from the off-gas composition sensors may negatively influence the still relatively low values of the consumption/production rates and hence the calculation of the carbon source feeding rate. For this reason, at the beginning of the fed-batch part, both feeding strategies (1 and 2) should only be used with caution and, preferably, alternative feeding strategies should be applied for the initiation of the fed-batch.

Similarly, in the first three experiments, the use of Strategy 1 after the onset of oxygen limitation, eventually led to the decrease in the DO level to zero, hence increasing the risk of carbon source overfeeding. Therefore, alternative feeding strategies were considered for the final phase of oxygen limitation.

Following the results obtained from the first three experiments, three auxiliary feeding strategies were evaluated as alternatives for the initial (Strategies 3,4 ) and closing (Strategy 5) stages of the fedbatch part. Furthermore, in the last experiment, also an alternative for the exponential growth phase was tested - Strategy 2 based on OUR.

The results from the final two experiments have shown that both feeding strategies based on production or consumption rates $(1,2)$ can be successfully replaced at the initial phase of the fedbatch part by continuous feeding following a linear feeding profile (Strategy 3) or semicontinuous feeding based on DO (Strategy 4). From the two, the latter is more reliable in terms of prevention of undesirable carbon source overfeeding, as it directly links the substrate dosage to the detection of carbon source limitation from the time course of the DO measurement. Similarly, in the final phase of oxygen limitation, the application of PID feedback control linking the carbon source feeding to DO regulation around a non-zero level (Strategy 5) ensures the prevention of overfeeding.

Finally, the last experiment (Cultivation 5) showed that for carbon source feeding in the exponential growth phase, an OUR-based strategy (Strategy 2) represents an equivalent alternative to the CPR-based approach (Strategy 1).

\section{Effect of feeding ratios on mcl-PHAs production}

As mentioned in the previous section, both strategies for continuous feeding proportional to CPR or OUR (Strategies 1,2) can be successfully applied for carbon source addition in the exponential growth phase. Hence, an important part of the experimental work was also the study of the effect of the ratio of the added carbon source (integral value over the exponential growth phase) to the amount of produced $\mathrm{CO}_{2}$ (integral value over the exponential growth phase), calculated by Eq. 6, i.e. Rat $_{\text {CXP POURCE STCORT }}^{\text {EXXXP PHASE END }}$ (it equals to $k C P R$ when Strategy 1 is used for carbon source feeding in the exponential growth phase, this was the case in Cultivations 1-4) or consumed $\mathrm{O}_{2}$, calculated by

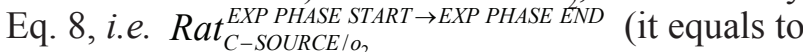
$k O U R$ when Strategy 2 is used for carbon source feeding in the exponential growth phase, this was the case in Cultivation 5), respectively, on the mclPHAs production. The obtained results indicate that by means of an appropriate choice of the ratio val- 


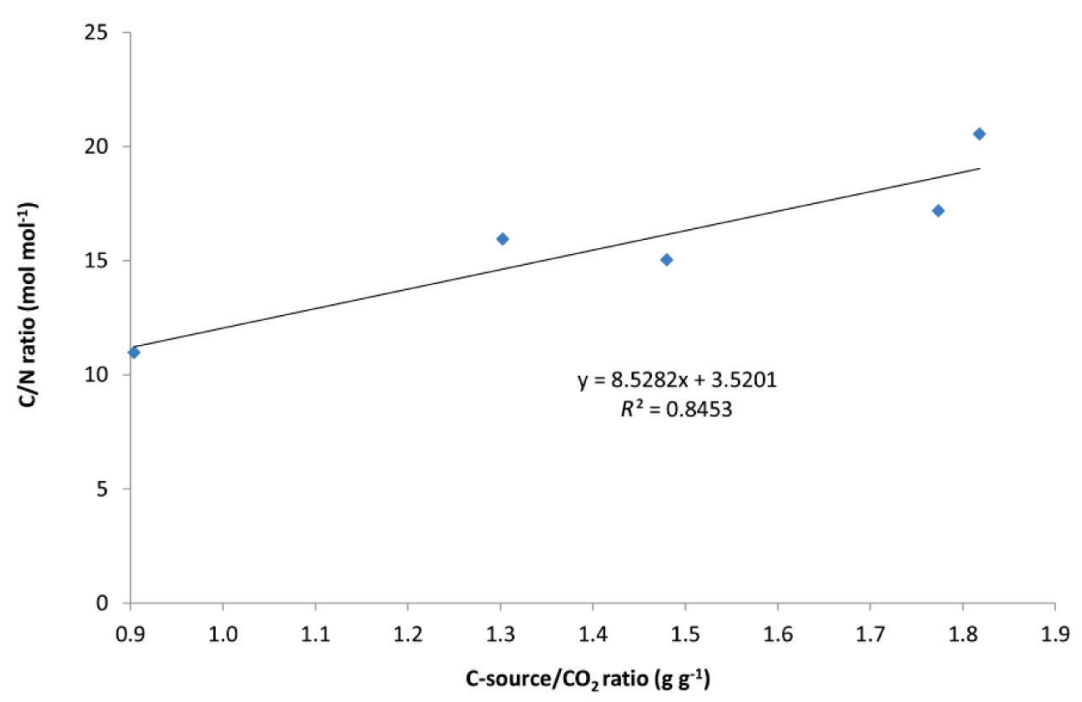

Fig. $4-\mathrm{C} / \mathrm{N}$ ratio in the feed vs. $\mathrm{C}$-source/CO${ }_{2}$ ratio in the exponential growth phase, data from cultivations $1-5$

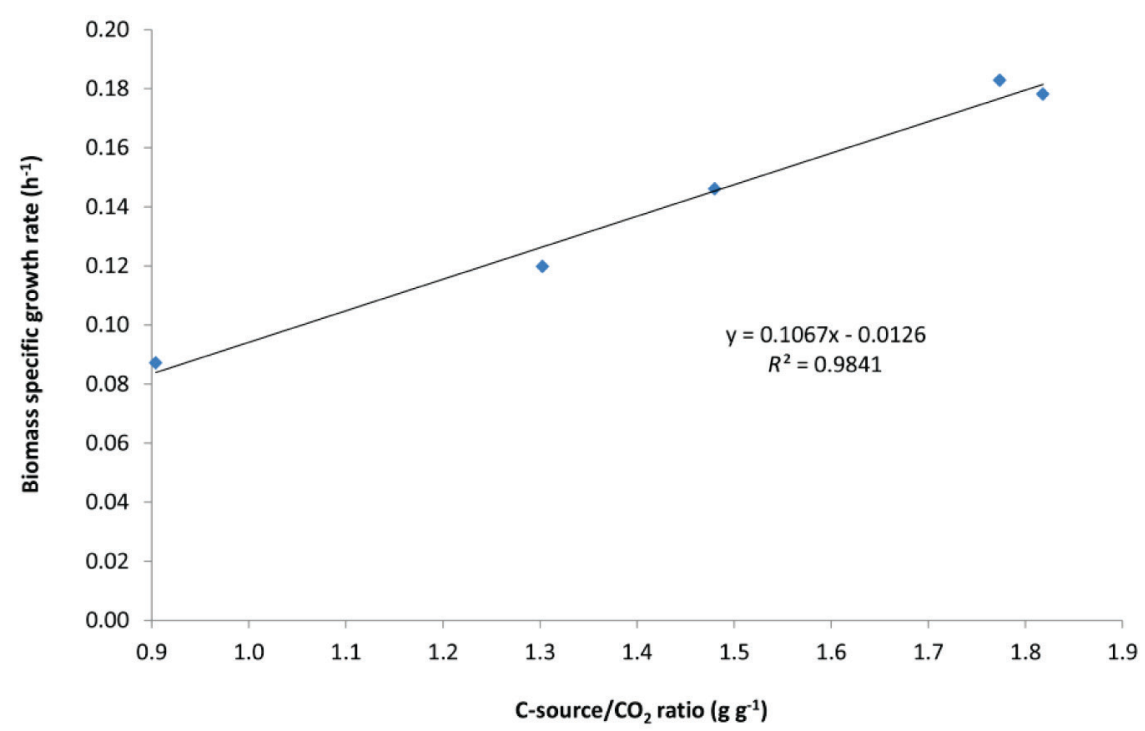

Fig. 5 - Overall specific biomass growth rate vs. $\mathrm{C}$-source/CO $\mathrm{C}_{2}$ ratio in the exponential growth phase, data from cultivations $1-5$

ue, it is possible to significantly influence the quality of the production process, as expressed by various indicators (e.g. overall specific growth rate of the biomass, productivity, conversion yield - see Table 1). More specifically, the concurrent use of $\mathrm{NH}_{4} \mathrm{OH}$ as base for $\mathrm{pH}$ control and octanoic acid as carbon source meant that, by changing the carbon source feeding ratio, it was possible to directly influence carbon/nitrogen $(\mathrm{C} / \mathrm{N})$ ratio in the feed (positive linear dependency, see Figure 4), which is well known to be an important factor in mcl-PHAs production ${ }^{12,13,14}$.

Likewise, a close positive linear correlation was also observed between the carbon source feeding ratios and the overall specific growth rate of the biomass (Figure 5).
On the other hand, the relation between the feeding ratios and indicators directly linked to mclPHAs production (PHA content, productivity, conversion yield) was found to be distinctly non-linear (Figures 6-8).

The highest values of these product-related indicators were obtained for $k C P R$ value of approx. $1.30 \mathrm{~g} \mathrm{~g} \mathrm{~g}^{-1}$ (the corresponding $k O U R$ value is ca. $1.05 \mathrm{~g} \mathrm{~g}^{-1}$, see Figure 3).

These conditions corresponded to the $\mathrm{C} / \mathrm{N}$ ratio in the feed of approx. $15 \mathrm{~mol} \mathrm{C} / \mathrm{mol} \mathrm{N}$ and the overall specific growth rate of $0.12 \mathrm{~h}^{-1}$. The obtained maxima of product-related indicators (max. PHA content, productivity) are comparable with the best results reported in the literature for the $P$. putida strains with octanoic acid as carbon source ${ }^{2,11}$. 


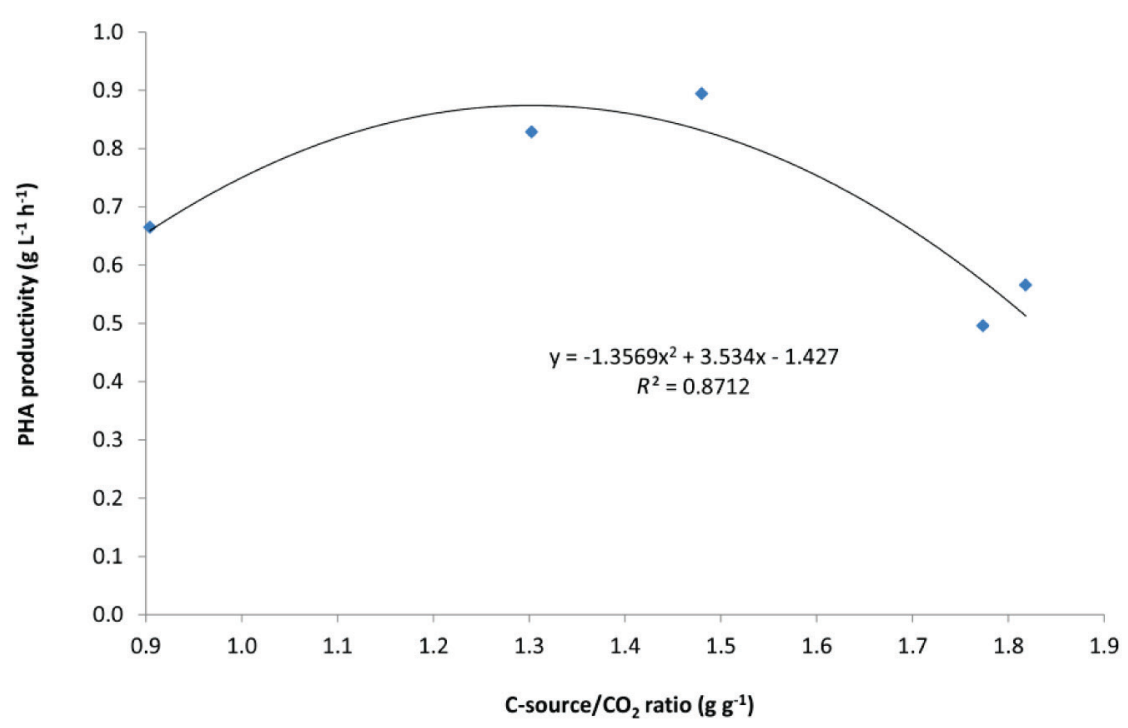

Fig. 6 - Mcl-PHAs productivity vs. C-source/CO $\mathrm{C}_{2}$ ratio in the exponential growth phase, data from cultivations $1-5$

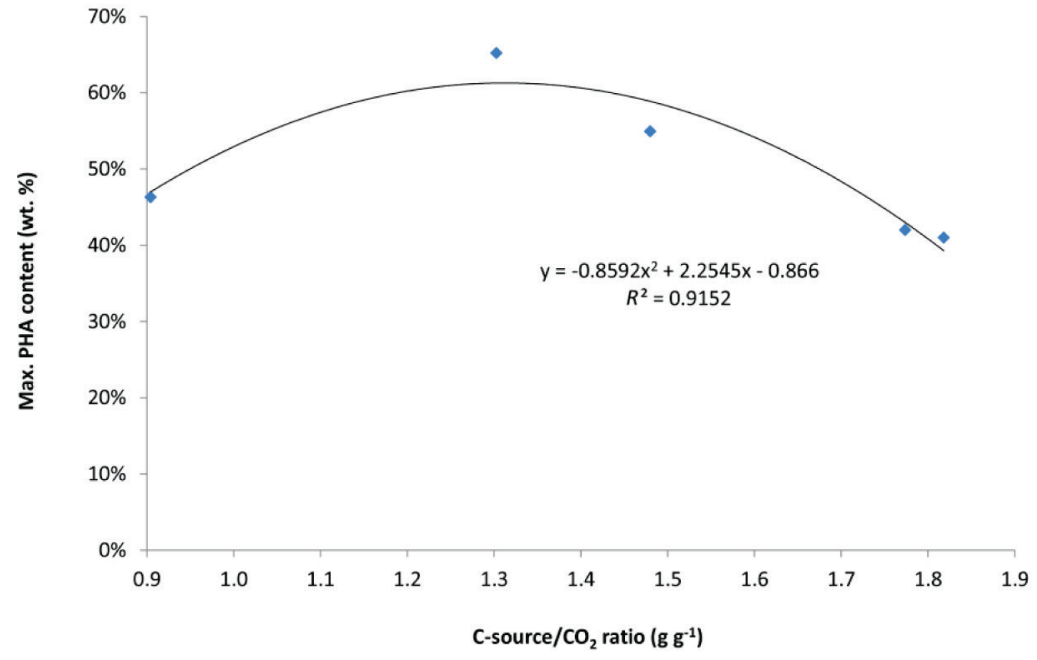

Fig. 7 - Max. mcl-PHA content vs. C-source/CO, ratio in the exponential growth phase, data from cultivations $1-5$

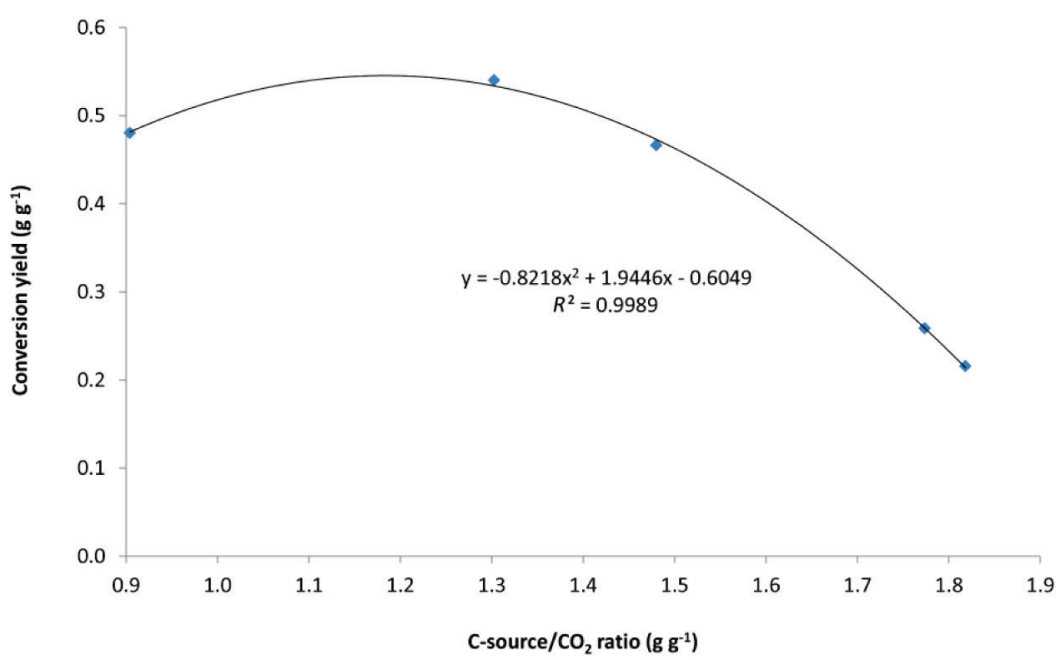

Fig. 8 - Conversion yield (C-source to mcl-PHAs) vs. C-source/CO $\mathrm{C}_{2}$ ratio in the exponential growth phase, data from cultivations $1-5$ 


\section{Conclusions}

A set of five feeding strategies (two main and three auxiliary strategies) have been proposed for a mcl-PHAs biopolymer production process (fedbatch cultivation of $P$. putida KT2442). The proposed strategies are suitable for fully automated dosage of carbon sources that are inhibitory at relatively low concentrations (octanoic acid in the presented study) for fed-batch cultivations of bacteria with physiological properties similar to those of $P$. putida KT2442. The strategies have been tested experimentally on a laboratory scale (7-L fermenter) in order to evaluate their performance and determine the proper sequence of their switching during the fed-batch part of the production process. Furthermore, in the case of the two main strategies (continuous feeding proportional to CPR or OUR, respectively), the optimal ratio (with regard to its effect on the biopolymer production) of the added carbon source to the amount of produced $\mathrm{CO}_{2}$ or consumed $\mathrm{O}_{2}$ has been determined.

The achieved results in terms of production indicators are fully comparable with the best results reported in the literature for this type of microbial strain (P. putida KT2442) and carbon substrate (octanoic acid).

The proposed feeding scheme (sequence of feeding strategies) is, unlike common feeding strategies based on predetermined exponential feeding profile, capable of taking into account the variability of individual bacterial cultures, because the carbon source feeding rate is being calculated online from process variables that reflect the actual state of the microbial culture. Furthermore, in the case of carbon source feeding in the exponential growth phase, the proposed $\mathrm{O}_{2}$ and $\mathrm{CO}_{2}$-based continuous feeding strategies provide an alternative method to established pulse-based DO-stat and $\mathrm{pH}$-stat feeding strategies, especially when uninterrupted feeding is the preferred carbon source feed mode.

\section{ACKNOWLEDGMENTS}

This work was supported by the 6th Framework Programme of the European Community under contract No. NMP2-CT-2007-026515 "Bioproduction project - Sustainable Microbial and Biocatalytic Production of Advanced Functional Materials" and by a specific university research (MSMT No 20/2015). This support is very gratefully acknowledged.

\section{References}

1. Koller, M., Salerno, A., Muhr, A., Reiterer, A., Braunegg, G., Polyhydroxyalkanoates: Biodegradable polymers and plastics from renewable resources, Mater. Tehnol. 47 (2013) 5.
2. Chen, G. Q., A microbial polyhydroxyalkanoates (PHA) based bio- and materials industry, Chem. Soc. Rev. 38 (2009) 2434. doi: https://doi.org/10.1039/b812677c

3. Sun, Z., Ramsay, J., Guay, M., Ramsay, B., Fermentation process development for the production of medium-chain-length poly-3-hyroxyalkanoates, Appl. Microbiol. Biotechnol. 75 (2007) 475. doi: https://doi.org/10.1007/s00253-007-0857-4

4. Kellerhals, M. B., Kessler, B., Witholt, B., Closed-loop control of bacterial high-cell-density fed-batch cultures: Production of mcl-PHAs by Pseudomonas putida KT2442 under single-substrate and cofeeding conditions, Biotechnol. Bioeng. 65 (1999) 306.

doi: https://doi.org/10.1002/(SICI)1097-0290(19991105)65: $3<306:$ :AID-BIT 8 $>3.0 . \mathrm{CO} ; 2-0$

5. Sun, Z., Ramsay, J., Guay, M., Ramsay, B, Automated feeding strategies for high-cell-density fed-batch cultivation of Pseudomonas putida KT2440, Appl. Microbiol. Biotechnol. 71 (2006) 423. doi: https://doi.org/10.1007/s00253-005-0191-7

6. Maclean, H., Sun, Z., Ramsay, J., Ramsay, B., Decaying exponential feeding of nonanoic acid for the production of medium-chain-length poly(3-hydroxyalkanoates) by $\mathrm{Pseu}$ domonas putida KT2440, Can. J. Chem. 86 (2008) 564. doi: https://doi.org/10.1139/v08-062

7. Sun, Z., Ramsay, J., Guay, M., Ramsay, B., Fed-batch production of unsaturated medium-chain-length polyhydroxyalkanoates with controlled composition by Pseudomonas putida KT2440, Appl. Microbiol. Biotechnol. 82 (2009) 657. doi: https://doi.org/10.1007/s00253-008-1785-7

8. Sun, Z., Ramsay, J., Guay, M., Ramsay, B., Enhanced yield of medium-chain-length polyhydroxyalkanoates from nonanoic acid by co-feeding glucose in carbon-limited, fedbatch culture, J. Biotechnol. 143 (2009) 262. doi: https://doi.org/10.1016/j.jbiotec.2009.07.014

9. Poblete-Castro, I., Rodriguez, A. L., Lam, C. M., Kessler, $W$., Improved production of medium-chain-length polyhydroxyalkanoates in glucose-based fed-batch cultivations of metabolically engineered Pseudomonas putida strains, J. Microbiol. Biotechn. 24 (2014) 59.

doi: https://doi.org/10.4014/jmb.1308.08052

10. Follonier, S., Panke, S., Zinn, M., A reduction in growth rate of Pseudomonas putida KT2442 counteracts productivity advances in medium-chain-length polyhydroxyalkanoate production from gluconate, Microb. Cell. Fact. 10 (2011) 1. doi: https://doi.org/10.1186/1475-2859-10-25

11. Carvalho Macário, V. F., Scale-up and design of chemometric models for the production of mcl-PHAs by Pseudomonas putida KT2442, MSc Thesis, Universidade Técnica Lisboa, (2009).

12. Huijberts, G. N. M., Eggink, G., Production of poly(3-hydroxyalkanoates) by Pseudomonas putida KT2442 in continuous cultures, Appl. Microbiol. Biotechnol. 46 (1996) 233. doi: https://doi.org/10.1007/s002530050810

13. Kim, B. S., Production of medium chain length polyhydroxyalkanoates by fed-batch culture of Pseudomonas oleovorans, Biotechnol. Lett. 24 (2002) 125. doi: https://doi.org/10.1023/A:1013898504895

14. Poblete-Castro, I., Escapa, I. F., Jäger, C., Puchalka, J., Lam, C. M., Schomburg, D., Prieto, M. A., Martins dos Santos, V. A., The metabolic response of P. putida KT2442 producing high levels of polyhydroxyalkanoate under single- and multiple-nutrient-limited growth: Highlights from a multi-level omics approach, Microb. Cell. Fact. 11 (2012) 1. doi: https://doi.org/10.1186/1475-2859-11-34 